\title{
Uveal tumour resection
}

\author{
Devron H Char, Theodore Miller, J Brooks Crawford
}

\begin{abstract}
Aim-To review the ocular retention rates, visual results, and metastases in uveal tumours managed with eye wall resection techniques.
\end{abstract}

Methods-This was a retrospective analysis of consecutive local uveal tumour resections performed by a single surgeon. All enucleation specimens were reviewed by one author. Both parametric and non-parametric analysis of data were performed.

Results-138 eyes were scheduled for eye wall resection surgery. The mean age was 52 years (range 11-86 years). Tumours involved predominantly the iris in 14 cases, iris-ciliary body in 57 , ciliary body alone in 18 patients, and in 49 cases the choroid was involved (ciliochoroidal, irisciliary body-choroid, or choroid). 125 eyes harboured melanomas; posterior tumours were more likely to have epithelioid cells $(p<0.05)$. The mean follow up was 6 years. The mean clock hours in iris and irisciliary body tumours was 3.5 . In tumours that involved the choroid the mean largest diameter was $12.9 \mathrm{~mm}$ and the mean thickness $8.5 \mathrm{~mm}$. 105 of $138(76 \%)$ eyes were retained. Histological assessment of surgical margins did not correlate evidence of tumour in enucleated eyes or metastatic disease. Surgical margins of more anterior tumours were more likely to be clear on histological evaluation $(p<0.05)$. Approximately $53 \%$ of retained eyes had a final visual acuity of $\geqslant 20 / 40$; visual results were significantly better in more anteriorly located tumours $(p<0.05)$. All retained iris tumour cases had $\geqslant 20 / 40$ final visual acuity. In tumours that involved the choroid nine of 31 retained eyes kept that level of visual acuity. Eight patients developed metastases; all metastatic events developed in patients with tumours that involved the choroid, and seven of eight were mixed cell melanomas. Conclusions-76\% of eyes were retained and $53 \%$ of these had a final visual acuity of $\geqslant 20 / 40$. Only $7 \%$ of uveal melanoma patients developed metastatic disease with a mean follow up of $\mathbf{6}$ years. Survival did not appear to be compromised with eye wall resection and in very thick, more posterior melanomas it appeared that ocular retention and visual results were better than with radiation alone. (Brf Ophthalmol 2001;85:1213-1219) California Pacific Medical Center, Davies Campus, 45 Castro Street, Suite 309, San Francisco, CA 94114, USA devron@tumori.org

Accepted for publication 2 April 2001
Attempts to remove uveal tumours with reten20th century. ${ }^{1}$ Zirm first reported a ciliary tion of the eye date from the first decade of the body resection in 1911 and Schubert resected a posterior tumour shortly thereafter. ${ }^{2}$ Vail summarised 244 reports before $1970 .{ }^{4}$

Many clinicians used iridocyclectomy technique to remove small anterior uveal tumours. ${ }^{4-11}$ A few pioneers, including Stallard, Foulds, and Peyman introduced techniques for resection of more posterior neoplasms. ${ }^{512} 13$ Our interest in ciliochoroidal resection techniques increased in the early 1980s when it became apparent that radiation of very thick, uveal melanomas, even with highly focused charged particles, had a very high incidence of post-treatment complications. ${ }^{14}$ As an example, ocular morbidity was approximately 10 times worse with irradiated tumours $\geqslant 8 \mathrm{~mm}$ thick than with those $\leqslant 5 \mathrm{~mm}$ thick. ${ }^{1}$

Several investigators, Foulds and Damato, Peyman and colleagues, Naumann and coworkers, Shields and collaborators, and us, have reported on modified techniques to resect uveal tumours. ${ }^{12}{ }^{15-18}$ Most have utilised a partial thickness scleral flap with various descriptive terms including cyclochoroidectomy, eye wall resection, choroidectomy, transscleral local resection, and partial lamellar sclerouvectomy. In a minority of cases either an en bloc scleral-uveal resection or an internal resection approach was performed, although the latter technique has been worrisome because of a higher theoretical concern about tumour dissemination. ${ }^{19} 20$

As we have accrued more data on uveal melanoma therapies our management patterns have evolved. We have continued to treat most posterior choroidal tumours or thinner anterior ciliochoroidal tumours with either helium ions or protons; thicker ciliochoroidal tumours and those involving the iris-ciliary body we have resected with adjunctive particle radiation for the posterior lesions. We present a retrospective series of uveal tumour resections from a single surgeon.

\section{Methods}

We reviewed 138 consecutive cases in which one surgeon (DHC) resected uveal tumours with intention to retain the eye. Surgeries were performed between 1977 and 2000 with follow up available to the end of July 2000. There was a slight female predominance with 74 females and 64 males. The mean age at surgery was 52 years (range 11-86 years). Tumour involved predominantly the iris in 14 cases, iris-ciliary body in 57 , ciliary body in 18 , ciliochoroidal in 38, iris-ciliary body-choroidal in four, and choroidal tumours alone in seven patients. The mean follow up was approximately 6 years (range 2 months to 23 years). 


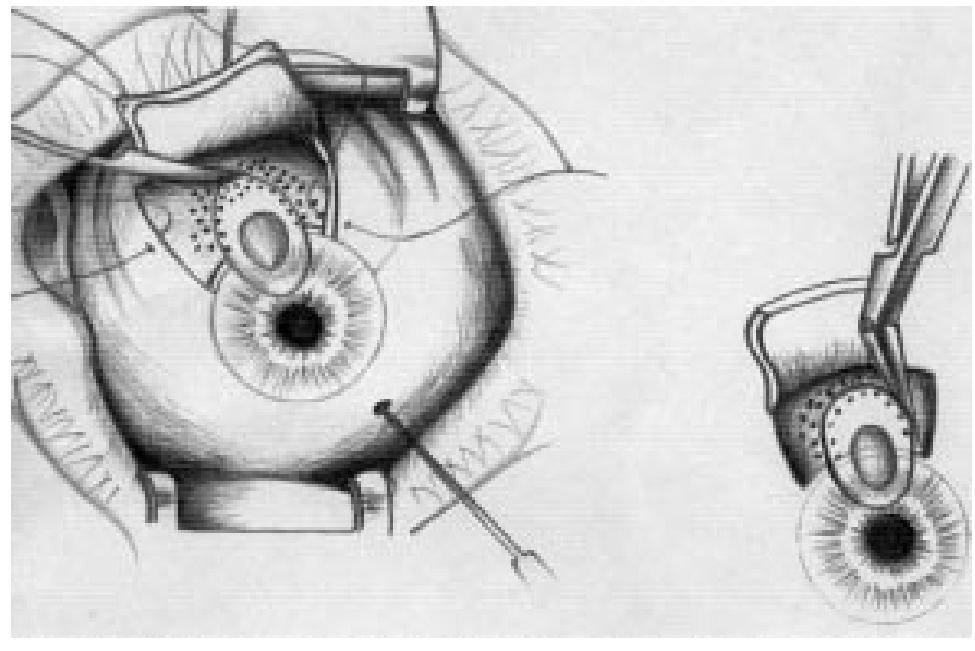

Figure 1 Schematic approach to resection of an iris ciliary-choroidal tumour.

Surgical techniques have previously been described. ${ }^{1}$ When medically not contraindicated, surgery was performed under hypotensive anaesthesia. Briefly, a modified 90\% thickness, posteriorly hinged, scleral flap was created, generally allowing $2-3 \mathrm{~mm}$ on each side of the tumour circumference determined by $180^{\circ}$ transillumination supplemented with direct tumour visualisation. A triple row of penetrating diathermy was placed around the sides and posterior edges of the resection bed. In posterior resections approximately $0.5-1 \mathrm{ml}$ of vitreous was aspirated through the pars plana approximately $180^{\circ}$ away from the tumour. The anterior incision was made through the limbus for predominantly iris or iris-ciliary body tumours. In almost all cases this incision was placed anteriorly so the angle structures could be removed as part of an en bloc resection. In cases in which we were suspicious of a possible ring melanoma (generally an apparently focal iris-ciliary body tumour with unilateral increased intraocular pressure), a fine needle aspiration biopsy (FNAB) was performed $180^{\circ}$ away from the mass. In posterior tumours an anterior incision was made approximately $2 \mathrm{~mm}$ in front of the anterior edge of the tumour. After the tumour was removed, if there was no vitreous loss, the incision was closed with 8-0 Vicryl sutures for the sclera and 10-0 nylon for the cornea. If vitreous loss occurred through the tumour incision, a vitrectomy through that site was performed. In tumours that involved less than 3 clock hours of iris, we often sutured closed the iris defect with an 11-0 proline suture. In ciliary body and posterior resections the vitreous base was supported with a No 42 encircling band. In a few patients with localised extrascleral extension, the sclera and uveal tissue were removed en bloc and the defect closed with a lamellar scleral graft taken from another quadrant of the eye.

In recent years, ciliochoroidal or choroidal tumours that were resected had tantalum marker rings placed approximately $2 \mathrm{~mm}$ outside of the tumour circumference at the time of surgery (see Fig 1) and these patients received postoperative helium ion or proton radiation to a tumour dose of $56 \mathrm{GyE}$, as described elsewhere.

Follow up information was obtained either from the surgeon's files or from the patient's current ophthalmologist. Follow up was obtained in over $95 \%$ of living patients. All histology was reviewed by one author (JBC) and all cytopathology by another author (TM). Statistical analysis was performed with parametric and non-parametric techniques as appropriate.

\section{Results}

We were able to retain 105 of 138 eyes. We classified seven enucleations as perioperative; these eyes were removed at surgery. The reasons for these perioperative enucleations were expulsive haemorrhage (two cases), and five cases in which a collar button portions of the melanoma had broken through Bruch's membrane falling into the vitreous as the base was removed. In 28 cases enucleations were performed more than 1 month after the initial surgery for clinical suspicion of residual or recurrent tumour (seven cases), or late complications of surgery in 21 patients (intractable proliferative vitreoretinopathy, pain, poor vision, phthisis, patient request, etc) The mean interval between eye wall resection and enucleation was 3 years if the immediate enucleations are excluded (range 0-11 years). In tumours that were enucleated more than 1 month after the initial surgery, tumour was noted in the eye specimen in 16 cases. Retinal detachments occurred either at the time or after surgery in 19 eyes. Nine of these cases were successfully repaired with good postoperative vision. The mechanism in retinal detachment was an obvious hole in the retina in six and traction or proliferative retinal vitreoretinopathy in the remainder.

In anterior tumours (iris-ciliary body or ciliary body) the mean number of clock hours involved was 3.5 (range 2-5 clock hours). In ciliochoroidal, choroidal, and iris-ciliary bodychoroidal tumours the mean diameters were $12.9 \mathrm{~mm} \times 10.4 \mathrm{~mm}$ (range $6-20 \mathrm{~mm}$ ) and a mean thickness of $8.5 \mathrm{~mm}$ (range $4.5-13.8$ $\mathrm{mm})$.

On histological review of the resected uveal tissue, 125 patients had uveal melanomas. There were 20 non-melanoma tumours, 10 of which were known to be non-melanomas before resection; the other 10 tumours had documented growth. The non-melanoma histologies are listed in Table 1.

There was a tendency for more posteriorly located uveal melanomas to have more epithelioid cells $(p<05)$. In iris melanomas nine of 11 were spindle cell type; two were mixed cell melanomas. In iris-ciliary body tumours 32 were spindle cell, 14 were mixed, and one was an epithelioid melanoma. In ciliary body

Table 1 Histology of non-melanoma cases

Melanocytoma

Atypical naevus versus low grade melanoma Metastatic tumour

Pigment epithelial adenoma

Conjunctival squamous cell carcinoma

Mesectodermal leiomyoma

Leiomyoma

Foreign body 
tumours, eight were spindle and five were mixed cell melanomas. In ciliochoroidal tumours 12 were spindle, 32 mixed, and one epithelioid cell melanoma. In melanomas that were either isolated to the choroid or were irisciliary body-choroidal tumours, all 11 were mixed cell melanomas.

The adequacy of surgical resection is often difficult to ascertain since there is differential shrinkage between a very thick uveal tumour and normal uvea placed in formalin. There were significantly more cases with positive tumour margins in posterior compared with anterior melanoma resections $(p<0.01)$. In predominantly iris tumours there were three cases with questionable margins and one with positive margins; two of these eyes (ring melanomas) were eventually enucleated and had tumour present in the enucleated specimen. In iris-ciliary body tumours there were 19 with positive margins and 11 with questionable margins; only 16 of these eyes have been enucleated; the others are doing well, without adjunctive radiation, with a mean follow up of over 6 years. In ciliary body tumours there were four with positive margins and two with questionable margins; two of three cases with positive margins were enucleated. The other cases are doing well, some with good vision and no sign of recurrence with a mean follow up of over 6 years. In the posterior lesions that involved the choroid there were 23 with positive margins and five with questionable margins. Nineteen eyes with tumours that involved the choroid have been removed; 13 had tumour in the enucleated specimen.

Since 1983, 18 ciliochoroidal or choroidal melanomas with either positive or questionable margins were treated with adjunctive proton or helium ion radiation. Only three of those irradiated eyes were subsequently removed (included in the cases described above); three did not have active tumour, two developed neovascular glaucoma and pain, and the other was enucleated by the referring physician because of concern about a recurrence, although no residual tumour was found.

In non-irradiated eyes with tumours in any location that were enucleated more than 1 month after uveal resection, eight of nine cases with positive margins had tumour noted in the enucleated specimen. Only four of these patients were thought to have recurrent tumour clinically. In the two enucleated patients with questionable margins at eye wall resection, both had tumour present in the enucleated specimen; one had a suspected recurrence. In the eight cases that had clear margins at uveal resection, six had tumour at enucleation, although this was only clinically suspected in four patients.

Visual results were significantly better in eyes with anteriorly resected tumours $(\mathrm{p}<0.01)$. The number of cases in this analysis was limited since enucleated eyes were excluded. We divided our cases into tumours that involved predominantly the iris, iris-ciliary body, ciliary body, or choroid (iris-ciliary body-choroid, ciliary body-choroid, or choroid). Final visual acuities were divided into
Table 2 Vision as a function of tumour location

\begin{tabular}{llll}
\hline Tumour location & $\geqslant 20 / 40$ & $20 / 60-20 / 200$ & $<20 / 200$ \\
\hline Iris & 12 & 0 & 0 \\
Iris-ciliary body & 28 & 5 & 13 \\
Ciliary body & 6 & 4 & 4 \\
Choroid involved & 9 & 8 & 10 \\
\hline
\end{tabular}

$>20 / 50,20 / 60-20 / 200$, and $<20 / 200$ (Table 2). Several of the patients with poor acuities are possibly correctable with cataract surgery, but that procedure has not been performed in all such cases. In iris tumours 12 of 14 had a final visual acuity of better than 20/50; two eyes were enucleated. In iris-ciliary body tumours the final vision in the resected eye was $>20 / 50$ in 28 of 44 retained eyes, 20/60 to 20/200 in five, and $<20 / 200$ in 13 . In patients with resected ciliary body tumours, six of 15 retained eyes had $>20 / 50$ acuity, four of 15 had $20 / 60$ to $20 / 200$ vision, and four were $<20 / 200$. In tumours that involved the choroid, nine of 31 retained eyes had a final visual acuity of $>20 / 50$, eight were $20 / 60$ to $20 / 200$, and 10 had a final acuity of $<20 / 200$. There was no difference in visual results between eyes with questionable, positive, or clear surgical margins.

Twenty seven patients have died; eight developed known uveal melanoma metastases and died from tumour related mortality. All of these metastatic cases involved the choroid (iris-ciliary body-choroidal or ciliochoroidal melanomas). Seven of eight had mixed cell melanomas; one had a spindle cell tumour.

\section{Discussion}

In this retrospective study we have shown that many uveal melanomas can be resected with ocular retention and in some preservation of good vision. Overall, we retained $76 \%$ of eyes and $53 \%$ of those eyes had a final visual acuity of $\geqslant 20 / 40$. Several issues can and cannot be addressed by the data we and others have accrued.

There is a paucity of data that compare the efficacy of various eye salvage therapies on survival. We previously reported on a randomised, dynamically balanced study of charged particle therapy compared with brachytherapy for uveal melanomas; better local control was noted with particles. $^{21}$ There are no similar randomised studies on local uveal melanoma resection. It is difficult to compare surgical series because often there is a marked difference in interseries tumour parameters; small anterior tumours have a very small metastatic rate, while larger ciliochoroidal melanomas have up to a $50 \% 5$ year tumour related mortality. ${ }^{1}$ In our study all the metastases occurred in eight tumours that involved the ciliochoroidal area of the eye. We observed a $7 \%$ metastatic rate comparable to that reported by Shields and colleagues of 5\%, although we had, on average, larger tumours. ${ }^{17}$ Similarly, Naumann and Rummelt noted a 91\% 10 year survival in 49 anterior uveal melanoma patients treated with a bloc sclera-uveal resection. ${ }^{19}$ Unlike some other reports with a median follow up of only 3 years, the mean follow up in our study was longer at 
approximately 6 years. In a retrospective, case matched study with 3 year follow up, Augsburger and associates compared 30 uveal melanoma patients treated with cobalt plaques versus 30 patients who had surgical resection. They noted a statistically insignificant better survival with resection, although these were, on average, smaller tumours than we treated. ${ }^{22}$ Damato and his Glasgow colleagues reported on metastatic patterns in 332 resected uveal melanomas. The tumours were as large as ours, but unlike both our data and data reported by Shields and colleagues, approximately two thirds were limited to the choroid. The absence of ciliary body involvement may have resulted in slightly better survival than in series with more ciliochoroidal tumours. ${ }^{1}{ }^{15} 17$ Damato and collaborators noted that factors associated with metastatic disease were similar to those observed with other therapies except that lack of adjunctive radiation and superior location also correlated with a worse prognosis. ${ }^{15}$ Metastatic deaths occurred in 52 of 332 $(16 \%)$; older patients, more malignant cell type, and largest tumour diameter were the factors most associated with metastases. ${ }^{15}$ Surprisingly, incomplete tumour resection and small persistent tumours were not significantly associated with metastases, although the median follow up was only 33 months in living patients. ${ }^{15} 16$

In both the Scottish and our experience, retrospective comparison of the survival of patients whose uveal melanomas were locally resected was no worse than those that were irradiated or enucleated. In some other series it is difficult to compare the metastatic rates either because of short follow up or tumours of very different prognoses. ${ }^{17}{ }^{20}$ As an example of the latter issue, in a series of internal resected neoplasms, over $90 \%$ were near the optic nerve or fovea with a mean tumour diameter of 8 $\mathrm{mm}$. The metastatic rate of $9.4 \%$ in that cohort is difficult to compare with our series with larger diameter, thicker tumours that involved the anterior choroid; all three of these factors are associated with worse prognosis. ${ }^{1} 20$

Accurate assessment of histological tumour margins is difficult for several reasons..$^{23}$ In our experience we found pathological assessment of tumour margins to be not as exact as we had hoped. Several eyes with anterior tumours (iris, iris-ciliary, and ciliary body melanomas) that we resected with either questionable or positive tumour margins and have been retained, often with good vision and no recurrence over 6 years after surgery. In tumours with either questionable or positive margins that involved the choroid, adjunctive radiation has been given. In three posterior melanomas treated before our routine use of radiation for questionable or positive margins, we were able to salvage small recurrences that developed 3-4 years later with radiation in two cases, and one with laser in one patient, with long term tumour free results.

We observed that tumour involvement of the resection margin was significantly more frequent in more posterior melanomas. In enucleated eyes almost all melanomas with positive tumour margins at the time of local resection had tumour noted in the enucleated specimen. Even in eight enucleated cases that had clear margins at initial tumour resection, six had tumour in the enucleated specimen. In one of these latter cases the margins in both the resection and enucleation specimens were clear, but tumour had developed in a different portion of the eye. All clinical recurrences were observed in the first 5 years after resection. It was disturbing to us that some late enucleations, performed for non-recurrence issues still had some residual tumour, although none of these patients have developed metastatic disease. All of these data make it mandatory that patients who have had local resections must be closely followed.

In some previous series of anterior melanoma resections the presence of positive tumour margins did not portend recurrence. Forest and colleagues reviewed 107 cases; most were small iris-ciliary body tumours less than 2 clock hours in extent. ${ }^{24}$ By current definitions of malignancy it is uncertain whether some of the 52 tumours categorised as spindle A melanomas would now be considered naevi. Only 32 of these iridocyclectomies had histological evidence of complete tumour excision; 68 had tumour cells extending to a margin, but only 12 recurred. ${ }^{24}$ In a smaller study, with almost one third of patients ineligible for study because of absent data and $53 \%$ of tumours being benign, Memmen and McLean reported that only three of 13 tumours with a significant number of epithelioid cells had clear margins. ${ }^{25}$ Malbran and colleagues noted that six of 10 tumours with positive margins recurred with a follow up as long as 11 years. ${ }^{26}$ Damato and colleagues reported 310 resections, approximately two thirds for choroidal melanomas, with a median follow up of 36 months. ${ }^{16}$ They noted that the histological assessment of tumour margins did not significantly correlate with recurrence; in their series, tumours closest to the optic nerve and fovea, larger diameter, more malignant cell type, and lack of adjunctive radiation had the highest chance for recurrence. ${ }^{16}$ Overall, they observed a 32\% incidence of recurrent disease. ${ }^{16}$ In a series from Philadelphia, $11 \%$ of cases recurred. ${ }^{17}$ In a series limited to 49 anterior uveal melanoma resections there was a 3\% incidence of local recurrence. ${ }^{17} \mathrm{We}$ observed that $9 \%$ of patients were thought clinically to have recurrent disease, but if all non-perioperative enucleations with tumour still present are also included, the incidence was $17 \%$. It remains uncertain in all of these series what the recurrence rate will be with longer follow up. As discussed above, several of our cases with positive margins and almost one half of similar cases described by Damato and colleagues were retained. Some patients may still develop recurrent tumour with longer follow up, since with radioactive plaques we have observed late reactivations as long as 10 years after treatment. $^{21}$

As discussed by Damato and Foulds, adjunctive radiation is correlated with both a lower incidence of recurrence and metastases. ${ }^{15}{ }^{16} \mathrm{In}$ 
melanomas that involve the choroid we now routinely use adjunctive radiation and hope that it will decrease recurrent disease. Robertson and colleagues have emphasised there can often be both intrascleral as well as contiguous uveal involvement by tumour, and radiation should minimise the impact of these microscopic tumour deposits. ${ }^{27}$

The reasons for our inability to predict some tumour behaviour on the basis of histological assessment of margins are uncertain. As mentioned, it is sometimes difficult, given the differential shrinkage of a very thick uveal tumour versus normal uvea, to assess the true surgical margin. Uveal melanomas usually arise from naevi and it is possible that many of the cells at the tumour periphery may be more benign; hence a remnant of naevoid cells would not be associated with either recurrence or metastases. It is also possible that there is an association between involvement of surgical margins and prognosis but we are not detecting it. The latter possibility could be due to either questions of power analysis or limited long term follow up of high risk cases. The number of eyes that have had resections with more malignant cell types with long follow up is limited. While several relatively large series have been reported, the situation could be analogous to that with the adverse effect of diabetic retinopathy on charged particle uveal melanoma radiation results-namely, until several hundred cases were available for analysis there were insufficient data to detect the effect. We doubt, analogous to eyelid basal cell carcinoma, that surgery induced inflammation and tumour destruction is an important mechanism of local tumour control in eyes retained with positive tumour margins.

The relative incidence of severe ocular morbidity, eye retention, and visual loss with various forms of uveal melanoma treatment remains unclear. Any retrospective surgical series, especially those that utilise a technically demanding procedure, have a significant learning curve. As surgeons have developed experience with these resection techniques, indications, contraindications, and complications have evolved.

Some early cases that we tried and failed to resect, we would no longer consider for resection. In a large ciliochoroidal tumour, hypotensive anaesthesia is required, and in the few patients in whom we attempted to resect, without this anaesthetic adjuvant we either failed with expulsive haemorrhages or severe haemorrhagic complications. We no longer attempt a posterior resection if the patient's cardiovascular status will not allow hypotensive anaesthesia. Damato and Foulds have emphasised that many small diameter thick tumours, especially with overlying serous detachment can be resected, and we agree. In very thick tumours that have broken through Bruch's membrane and invaded the retina, our results were dismal. In a few cases we were able to resect the tumour base, only to have the portion that eroded through Bruch's membrane fall off into the mid-vitreous mandating enucleation. As Foulds has pointed out, perhaps counterintuitively for the novice, tumour involvement of the ciliary body is associated with a higher complication rate..$^{12} 162728$

The incidence of complications depends on how they are defined and the size and location of the tumours. In a relatively early series, Kara noted that approximately $30 \%$ of mainly anterior tumour eyes developed complications. ${ }^{29}$ Shields and colleagues described over an $80 \%$ incidence of vitreous haemorrhage. ${ }^{17}$ An immediate or delayed haemorrhage into the vitreous is almost routine in any ciliary or choroidal resection. Fortunately, less than $10 \%$ of these cases require vitrectomy to clear the ocular media. Similarly it is difficult to determine the effect of these procedures on lens opacification. Very rarely the lens can be inadvertently damaged at surgery or sacrificed if a complex retinal repair is needed postoperatively. Many patients with these tumours present with focal lens opacities as diagnostic components that lead the patient to seek care. ${ }^{1}$ Fortunately, in over $90 \%$ of cases in which we later have done cataract surgery the results of that procedure were excellent. Proliferative vitreoretinopathy, cystoid macular oedema, and phthisis are the most serious sequelae of uveal tumour resections. Most of the visual loss we encountered was because of these problems, and 21 of 28 late enucleations ( $>1$ month following the uveal resection) were done because of these problems or variations of them. Surprisingly, we did not see a significant difference in the incidence of complex retinal detachments in ciliary body compared with choroidal resections.

Visual results are difficult to compare in different series. Overall, over $53 \%$ of our retained eyes kept good vision. As we expected there were statistically significantly better visual results in anterior compared with posterior tumours $(\mathrm{p}<0.01)$. In predominantly iris tumours, all retained eyes had $\geqslant 20 / 40$ final acuity. In high risk-ciliochoroidal melanomas where we probably would have had an equal incidence of enucleations after particle radiation because of a mean tumour thickness of 8.5 $\mathrm{mm}$, the visual results were slightly better than in our irradiated cohort; nine of 31 eyes retained $\geqslant 20 / 40$ visual acuity after resection. It is difficult to compare visual results in different series for several reasons: different surgical techniques, different tumour locations and different tumour sizes. In a clinicopathological report, Memmen and McLean noted that only six of 23 anterior tumour patients retained $>20 / 60$ vision. ${ }^{25}$ In 30 resected cases reported by Augsburger and co-workers, with a median follow up of 36 months; two thirds had visual acuities of $<20 / 200 .^{22}$ Peyman and colleagues observed that $31 \%$ of eyes retained better than $20 / 200$, although these tumours were near the optic nerve and/or fovea. ${ }^{20}$ In anterior tumours Naumann and Rummelt noted 53\% had 20/60 final visual acuity. ${ }^{17}$ As Damato and co-workers emphasised, visual results are best in small tumours that do not involve the ciliary body and are distant from the optic nerve and fovea. ${ }^{28}$ While we agree with that statement, a caveat should be added. In our experience the 
ability to predict visual outcome in tumours involving the ciliary body or choroid was not precise; some eyes, even with relatively small tumours had very poor visual outcome, despite no obvious problems noted at the time of surgery.

All of the series described emphasise a point we have previously made about the inaccuracy of non-invasive diagnosis, especially for anterior lesions. ${ }^{30}$ While in eyes enucleated in major centres with the clinical diagnosis of uveal melanoma the diagnostic accuracy is almost $100 \%$; the accuracy in smaller centres, especially of peripheral tumours is less. ${ }^{30}{ }^{31}$ In our series, $14 \%$ of resected tumours were not uveal melanomas. In several of these cases we were aware of that fact on the basis of FNAB, but felt a growing tumour should be removed. In approximately one half of these cases we were not aware that the tumour was not a melanoma until we examined it histologically. In almost all of these we were going to resect the tumour regardless of cytopathology data and did not perform a FNAB. In most of the other series reported, there has similarly been between $15 \%$ and $30 \%$ incidence of resections for nonmelanomas, although some reports have had even a higher number of simulating lesions. ${ }^{4} 1720$ 32-41

The choice of radiation, enucleation, or local uveal resection is sometimes arbitrary. We believe that there are sufficient data to at least explain or advocate the use of one or the other of these modalities in several settings. Both the group in Philadelphia and we have tended to use radiation therapy for most posterior and peripheral uveal melanomas less than $6 \mathrm{~mm}$ in height. ${ }^{1}$ Our rationale for this choice is threefold. One, radiation has had a higher local control rate; in our charged particle data we have had $>97 \%$ local control; no series of surgical resections has as high a control rate. ${ }^{21}$ Two, many uveal melanomas are diagnosed in older, often hypertensive patients; in such cases there probably is less systemic damage with a short procedure to place either tantalum marker rings for proton irradiation or place a radioactive plaque compared with a surgical procedure under hypotensive anaesthesia that usually is three to five times longer in duration. Three, in posterior tumours near the disc and fovea, as Damato and Foulds have pointed out, there is a threefold higher recurrence rate. In the series of posterior uveal tumours reported by Peyman's group the visual acuity was $\geqslant 20$ / 200 in only $31 \%$, a figure comparable to radiation. $^{20}$ In a setting where we can retain approximately $90 \%$ of irradiated eyes and surgery is much less taxing for the patient we believe radiation is a better option both for vision and local tumour control. ${ }^{42}$

In contrast, in very thick tumours, independent of location there is a much higher incidence of visually destructive radiation complications. ${ }^{42}$ Especially in such thick ciliochoroidal or choroidal melanomas we believe that there may be a better result with resection, although as our series points out there is significant morbidity associated with either approach. In very thick ciliochoroidal tumours that were treated with charged particles alone, we noted that $>50 \%$ developed significant complications, and many of these eyes were lost. ${ }^{42} \mathrm{We}$ did have to remove 25 of 49 melanomas that involved the choroid in this series, although it is important to emphasise that these were large tumours with a mean diameter of over $12 \mathrm{~mm}$ and a thickness of $8.5 \mathrm{~mm}$.

At present we are able to retain over $80 \%$ of all uveal melanoma eyes we manage by all methods. ${ }^{1}$ As this series and others demonstrate, despite many caveats, survival does not appear compromised with these uveal resection techniques; many eyes are salvaged that would be lost with other therapies.

A portion of this report was presented at the American Ophthalmological Society meeting in May, 2000

1 Char DH. Tumors of the eye and ocular adnexa. Toronto: BC Decker, 2001

2 Zirm E. Über endobulbare operationen. Arch Augenheilkd 1911;69:233-51.

3 Schubert F. Operation eines leucosarkomas der choroidea mit Erhaltung des Auges. Wien Klin Wochenschr 1925;38: 677-8.

4 Vail DT. Iridocyclectomy, A review. Am f Ophthalmol 1971; $71: 161-8$

5 Stallard HB. Partial cyclectomy. Br F Ophthalmol 1964;48:1-

Winter FC. Surgical excision of tumours of ciliary body and iris. Arch Ophthalmol 1963;70:19.

7 Cleasby GW. Malignant melanoma of iris and ciliary body: surgical excision. Trans Am Acad Ophthalmol Otolaryngol

8 Borley WE, Miller WW. Iridocyclectomy. Am f Ophthalmol 1965;60:829.

9 Muller HK, Sollner F, Lund OE. Erfahrungen bei der Muller HK, Sollner F, Lund OE. Erfahrungen bei der
operativen Entfernung von Tumoren der Iriswurzel und des Ciliarkorpers. Deutsch Ophthal Ges 1963;63:194.

10 Sears ML. Technique for iridocyclectomy. Am $\mathcal{f}$ Ophthalmol 1968;66:42.

11 Sugar HS. Posterior lamellar resection of cornea for epithelial implantation cyst in anterior chamber. Am $\mathcal{F}$ Ophthalmol 1962;54:800

12 Foulds WS. The local excision of choroidal melanomata. Trans Ophthalmol Soc UK 1973;93:343-6.

13 Peyman, Juarez CT, Diamond JG, et al. Ten years' experience with eyewall resection for uveal malignant melanomas. Ophthalmology 1984;91:1720-5.

14 Char DC, Saunders W, Castro JR, et al. Helium ion therapy for choroidal melanoma. Ophthalmology 1983;90:1219-25.

15 Damato BE, Paul J, Foulds WS. Risk factors for metastatic uveal melanoma after trans-scleral local resection. Br $\mathcal{F}$ uveal melanoma after trans-sc
Ophthalmol 1996;80:109-16.

16 Damato BE, Paul J, Foulds WS. Risk factors for residual and recurrent uveal melanoma after trans-scleral local resecrecurrent uveal melanoma after trans-s

17 Shields JA, Shields CL, Shah P, et al. Partial lamellar sclerouvectomy for ciliary body and choroidal tumors. Ophthalmology 1991;98:971-83.

18 Char DH. Choroidal melanomas:alternative therapies. Trans Pacific Coast Oto-Ophthalmol Soc 1980;60:9-14.

9 Naumann GO, Rummelt V. Block excision of tumors of the anterior uvea. Report of 68 consecutive patients. Ophthalmology 1996;12:2017-27.

20 Kertes PJ, Johnson JC, Peyman GA. Internal resection of posterior uveal melanomas. $\mathrm{Br} \mathcal{F}$ Ophthalmol 1998;82: $1147-53$.

21 Char DC, Quivey JM, Castro J, et al. Helium ions versus iodine 125 brachytherapy in the management of uveal melanoma: a prospective randomized dynamically balanced trial. Ophthalmology 1993;100:1547-54.

22 Augsburger JJ, Lauritzen K, Gamel JW, et al. Matched group study of surgical resection versus cobalt-60 plaque
radiotherapy for primary choroidal or ciliary body melanoma. Ophthalmic Surg 1990;21:682-8.

23 Margo CE. Microscopic examination of surgical margins of intraocular tumors excised en bloc. Ophthalmol Surg Laser 1998; 29:692-4.

24 Forrest AW, Keyser RB, Spencer W. Iridocyclectomy for melanomas of the ciliary body: a followup study of pathology and surgical morbidity. Trans Am Acad Ophthalmol Otolaryngol 1978;85:1237-49.

25 Memmen JE, and McLean IW. The long-term outcome of patients undergoing iridocyclectomy. Ophthalmology 1990; 97:429-32.

26 Malbran ES, Charles D, Garrido CM, et al. Iridocyclectomy technique and results. Ophthalmology 1979;86:1048-66.

27 Robertson DM, Campbell RJ, Weaver DT. Residual intrascleral and intraretinal melanoma: a concern with lamellar sclerouvectomy for uveal melanoma. Am f Ophthalmol 1991;112:590-3.

28 Damato BE, Paul J, Foulds WS. Predictive factors of visual outcome after local resection of choroidal melanoma. $\mathrm{Br} \mathcal{F}$ Ophthalmol 1993;77:616-23. 
29 Kara GB. Excision of uveal melanomas: a 15 year experience. Trans Am Acad Ophthalmol Otolaryngol 1979; 36:997-1023

30 Char DC, Miller T. Accuracy of presumed melanoma diagnosis prior to alternative therapy. Br $\mathcal{F}$ Ophthalmol 1995;79: 692-6.

31 Char DH, Stone RD, Irvine AR, et al. Diagnostic modalities in choroidal melanoma. Am F Ophthalmol 1980;89:223-30.

32 Volkov VV. Indications, technique and results of surgical treatment of intraocular melanomas. In: Lommatzsch PK, Blodi FC, eds. Intraocular tumors. Berlin: Akademie-Verlag, 1983:378-85

33 Naumann GOH. Block excision of tumors of the ciliary body and choroid. In: Lommatzsch PK, Blodi FC, eds. Intraocular tumors. Berlin: Akademie-Verlag, 1983:386-95.

34 Brovkina AF. Surgical treatment of tumors of the anterior uvea. In: Lommatzsch PK, Blodi FC, eds. Intraocular uvea. In: Lommatzsch PK, Blodi FC, eds.
tumors. Berlin: Akademie-Verlag, 1983:404-8.

35 Busse H, Juneman G, Kroll P, et al. Technik und Ergebnisse, der Iridozyklektomie. Klin Monatsbl Augenheilkd 1986;188: der Iridozy.
36 Peyman GA, Schulman J, Raichand M. Diagnosis and therapeutic surgery of the uvea-Part 2: results. Ophthal Surg 987;18:310-16.

37 Farber MG, Smith ME, Gans LA. Astrocytoma of the ciliary body. Arch Ophthalmol 1987;105:536-7.

38 Ainsworth JR, Damato BE, Lee WR, et al. Follicular thyroid carcinoma metastatic to the iris:a solitary lesion treated with iridocyclectomy. Arch Ophthalmol 1992;110:19-20.

39 Naumann GOH, Rummelt B. Block excision of cystic and diffuse epithelial ingrowth of the anterior chamber. Arch Ophthalmol 1992;110:223-7.

40 Shields JA, Shields CL, Eagle RC, et al. Observations on seven cases of intraocular leiomyoma. Arch Ophthalmol 1994;112:521-8.

41 Spraul CW, d'Heurle D, Grossniklaus HE: adenocarcinoma of the iris tumor epithelium. Arch Ophthalmol 1996;114: $1512-7$

42 Char DH, Kroll SM, Castro J. Ten-year follow-up of helium ion therapy for uveal melanoma. Am $\mathcal{f}$ Ophthalmol 1998;125:81-9. 